\title{
Image-guided therapies for prostate and kidney cancers
}

\author{
Rafael Sanchez-Salas ${ }^{1} \cdot$ Mihir Desai $^{2}$
}

Published online: 27 February 2019

(c) Springer-Verlag GmbH Germany, part of Springer Nature 2019

A particularized and more rational treatment for both prostate and renal carcinomas has been introduced and refined following the great development of Imaging in the medical field. These options remain both experimental and controversial; the aim of this topic issue was to clearly state the role of imaging guided ablative techniques for the treatment of prostate and renal cancer [1].

The quest for an effective and less aggressive treatment for prostate and renal tumours is not new. In the prostate side, we have witnessed an enormous evolution from open surgery to classic and robot-assisted laparoscopic techniques that completely changed the evaluation of outcomes in prostate cancer and positively impacted the morbidity and quality-of-life results of the surgical intervention. Radiotherapy has also evolved to remain cancer-control effective with less associated collateral damage. Barret et al. (on this issue), addressed the essential role of multiparametric MRI in the Prostate Cancer diagnostic pathway. Imaging has been broadly embraced by the urological community and a great deal of information has to be taken into account for diagnosis, treatment, and follow-up. Futhermore, the idea of having a detailed information on an eventual index lesion within the prostate, has completely changed prostatic biopsies, which evolved from a "blind approach" to a more detailed and comprehensive technique, aiming to diagnose those lesions actually needing some kind of treatment. Konety et al. (on this issue) present the different available options for prostatic biopsies, as well as, the real benefits obtained with the more recent MRI-guided approach.

The possibility of diagnosing a region of interest within the prostate has further developed the field of prostatic ablative treatments. Several energies are available and recent

Rafael Sanchez-Salas

rafael.sanchez-salas@imm.fr

1 Department of Urology, L'Institut Mutualiste Montsouris, Paris, France

2 Center for Advanced Robotics, Keck School of Medicine of USC, Los Angeles, USA development aims to provide partial gland ablation rather than the whole gland approach. Lodeizen et al. (on this issue), clearly present the available energies for prostatic treatment, as well as, their mechanisms of action, performance and limitations. The field of Focal Therapy for Prostate Cancer represents a paradigm shift on the Prostate Cancer arena and although still experimental, it holds the potential to become the middle ground option between active surveillance and more radical options. Oncologic follow-up for these patients is of utmost importance and Tay Jack et al. (on this issue) present a clear picture of how the evaluation of outcome should be undertaken with these patients [2].

In kidney cancer the wave of improvement has been similar. From the original open procedures offered to patients in the past, we have moved forward to a cancer-control safe and reliable minimally invasive nephron-sparing renal surgery.

Simultaneously, we have become aware of the indolent nature of some kidney and prostate tumours that can eventually undergo surveillance based on patient's particular characteristics. Furthermore, several needled-based ablative techniques have been coined to effectively treat tumours in a less invasive fashion. Jewett et al. (on this issue) show the importance of a renal biopsy on the decision making for a small renal mass approach. Their manuscript features indications and outcomes of the technique. Zondervan et al. (on this issue) present the available energies that could be deployed for renal mass ablative treatment, their manuscript also features new available techniques for this approach.

Today, the cancer diagnostic and treatment pathways for both prostatic and renal carcinomas feature different therapeutical options. Focal therapy should be strongly supported on high-quality imaging: cutting-edge radiological technology and above all, urological dedicated radiologists able to reliably obtain the adequate information from their imaging work-up which is, tumours are to be identified and biopsied with imaging and the actual ablative treatment and follow-up control is also to be supported by these techniques.

Urology has always been a technologically bound surgical specialty. As the Imaging tools have become of utmost importance to diagnose and treat urological malignancies, 
the urologist's role is to remain the cornerstone of the multispecialty team involved in the assessment of the disease.

This topic issue arises from an effort from a large international faculty, working in nine committees, as part of the ICUD-SIU on Image-guided therapies for prostatic and renal carcinomas held in Melbourne, Australia in October 2015 [3].

This project attempts to add a valuable piece of information on the subject of image-guided therapies for prostate and renal tumours through the academic philosophy of previous International Consultation on Urological Diseases initiated and promoted by Dr. Paul Abrams since 1993.

\section{References}

1. Marra G, Gontero P, Valerio M (2016) Changing the prostate cancer management pathway: why focal therapy is a step forward. Arch Esp Urol 69:271-280
2. van der Poel HG, van den Bergh RCN, Briers E et al (2018) Focal therapy in primary localised prostate cancer: the European Association of Urology position in 2018. Eur Urol 74(1):84-91

3. Sanchez-Salas R, Desai M (eds) (2015) Guided therapies for prostate and kidney cancers. A joint SIU-ICUD international consultation. Societé Internationale d'Urologie (SIU). ISBN: 978-0-9877465-9-7

Publisher's Note Springer Nature remains neutral with regard to jurisdictional claims in published maps and institutional affiliations. 